\title{
COMPLEXITY IDEAS FROM CONDENSED MATTER AND STATISTICAL PHYSICS
}

\author{
Luciano Pietronero,
}

Dipartimento di Fisica, Università di Roma La Sapienza e Istituto dei Sistemi Complessi, CNR Roma

Webpage: http://pil.phys.uniroma1.it

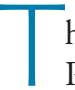
his field of physics was originally identified as Solid State Physics. Later P.W. Anderson coined the term Condensed Matter Physics and more recently it has merged with Statistical Physics to lead to the Physics of Complex Systems.

This area of physics is in a way complementary to that of elementary particles which is based on a reductionistic approach. The traditional approach of physics in fact, is to consider the simplest systems and study them in great detail. This approach focuses on the elementary "bricks" which are the building blocks of matter. This reductionistic vision can be applied to a great deal of situations and it implies necessarily the existence of characteristic scales: the size of an atom, of a molecule or of some macroscopic object.

On the other hand, there are many situations in which the knowledge of the individual elements is not sufficient to characterize the properties of the whole system. In fact when many elements interact in a nonlinear way, they can lead to complex structures which cannot be directly related to the individual elements. In these cases we can think at a sort of "Architecture" of nature, which depends in some way on the individual elements but, in addition, it manifests properties and fundamental laws which cannot be deduced from the individual elements. This point of view was first exposed in a famous article of P.W. Anderson (PWA) which had a deep impact in the development of complexity ideas.
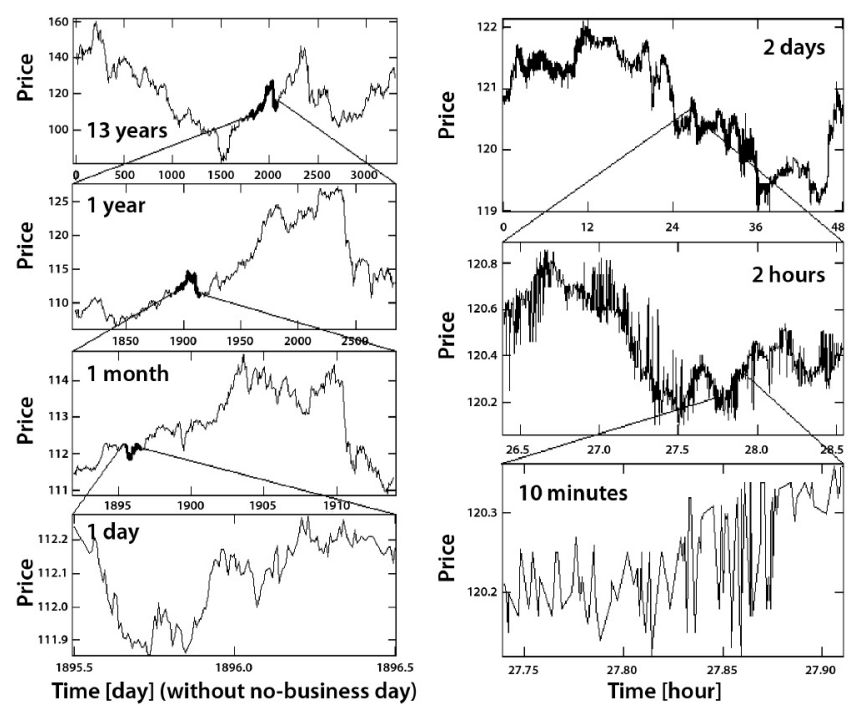

$\Delta$ FIG. 1: Example of scale invariance in financial data.

The exchange rate Yen/USD is plotted for various time scales. The top left panel refers to 13 years, the next panel to one year, then one month, one day and so on up to seconds. Up to the time scale of hours, the panels show a similar behavior (self-similarity) which changes drastically at very short times (Courtesy of H. Takayasu e M. Takayasu).

\section{More is different}

This is the title of the famous article of PWA (Science 177, 393, 1972) which is considered a sort of manifesto of Condensed Matter physics first, and later of Complexity. This article had a deep influence on the way scientists think about the problems they consider. In this paper Anderson questions the generality of the reductionist point of view according to which the fundamental laws are only those associated to elementary particles. The basic idea is that nature is organized in a hierarchical way and that there are individual elements and collective emergent properties every time one moves from a level of the hierarchy to the next one. The later development of the renormalization group has provided a formalism which permits to interpret these intuitions within a rigorous framework. Examples of these various levels can be quarks and nuclear physics, atoms, molecules, proteins, the emergence of life and on up to the macroscopic scales and the entire universe. The idea is that each discipline refers to the step between one level and the next one. In this process the essential concepts are the basic elements and their interactions. These lead to emergent properties and collective behaviours which cannot be identified from the original elements. From these collective properties one can then identify the basic elements of the next level of the hierarchy. Each of these steps is characterized by its own fundamental laws. In the recent years there have been many attempts to apply this reasoning also to fields which may appear very far from physics like socioeconomic disciplines.

In a Conference in Aspen in January 2000 Anderson recalls the origin of this paper. At the end of the sixties, in the political and economic area, various slogans appeared like "Small is beautiful" and "More is worse" of the British environmental movement. The establishment replied with "More is better" and, according to Anderson, it was natural to suggest that simply "More is different". The article originated from a sort of resentment that physicists in the field of condensed matter developed with respect to a certain arrogance of the field of elementary particles. For example V. Weiskopf (first CERN director) divided science between "intensive" (elementary particles) and "extensive" (all the rest). This vision implied the concept that elementary particle physics would be the only real intellectual challenge and the other fields would be "just chemistry". This hierarchical vision was accepted in some sense by Anderson but he subverted its terms: one cannot assume that the laws of a given science are just consequences of those of another one. The technical example with which this thesis was presented was that of the symmetry breaking and the properties of the superconductive state. 
This example shows that radically new properties and concepts can emerge from a relatively simple substrate. In fact the problem of superconductivity had challenged the best theoretical physicists of the first half of the $20^{\text {th }}$ century like Bloch, Wigner and Heisenberg who, however, moved along wrong directions. Some of them even thought that the solution would be impossible. The problem was finally solved by the "phenomenologist" John Bardeen with an intuition about the nature of the state which could not be derived with the traditional methods, however sophisticated.

The concept of "emergence" was instead borrowed from biology in relation to the emergence of life from unanimated matter. This concept was then exported in the field of physics and generalized. The subsequent concepts of the renormalization group and the associated properties of universality have then provided a formalism for this new vision. Universality, in fact, corresponds to the simplest way to demonstrate that the same macroscopic results can be obtained from widely different microscopic causes. The process on which this phenomenon is based is the spontaneous symmetry breaking. All the properties that characterize solids - like the crystalline structure, the metallic or insulating properties, elasticity and macroscopic coherence - are meaningless in a world of individual atoms. On the opposite they develop naturally as emerging properties of a systems with many atoms interacting among them.

This process reaches its completeness only if the number of particles is very large. In nuclei, which typically contain only about a hundred nucleons, there are elements of geometric structure and superfluidity. But, given the limited number of components, the process remains incomplete, because these properties are well defined only for an infinite number of particles.

In summary, the aim was to demonstrate the intellectual autonomy of the higher level phenomena with respect to the "tyranny" of the fundamental equations which were supposed to constitute the "theory of everything" (a term introduced later).

Also in the case of deterministic chaos one can observe, even in a classical system, a substantial decoupling between the initial conditions and the evolution of the system.

In conclusion: there are no absolute fundamental laws which, starting from the smallest scale, permit (at least in principle) the derivation of all the other properties at all the other scales. There are different levels and fundamental laws for each of them which permit the step to the next level. In this perspective the various scientific disciplines become part of the same global system with much more possibilities to be integrated among them.

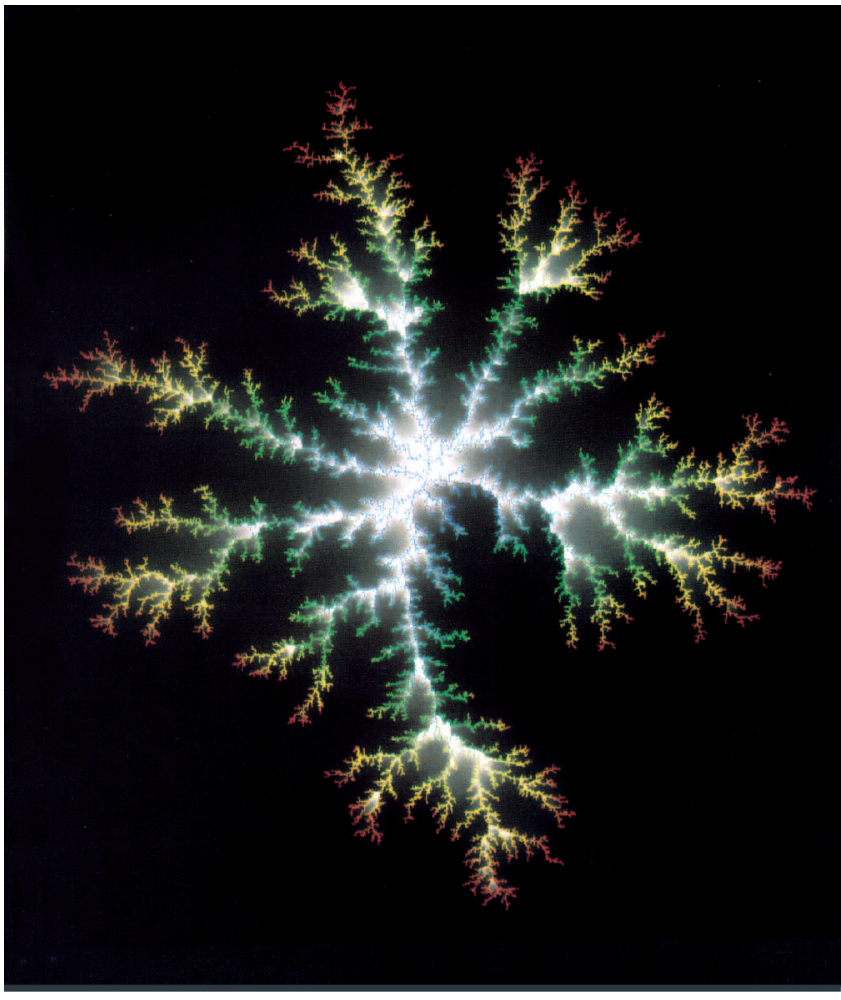

$\Delta$ FIG. 2: Aggregate generated by Laplacian growth process (Diffusion Limited Aggregation or Dielectric Breakdown Model).

A classical example of self-organized fractal growth dynamics corresponding to an irreversible process in which the growth probability is proportional to the gradient of the laplacian field around its contour. Given the generality of the Laplace equation this process captures the properties of several, apparently different, phenomena.

V FIG. 3: Cosmic complex structures.

Galaxy distribution in three dimensional space from the recent SLOAN data. Larger sample volumes constantly show larger correlated structures challenging the traditional conjecture of a homogeneous distribution of matter. Recently also the dark matter (lensing effect) shows complex structures analogous to those of visible matter.

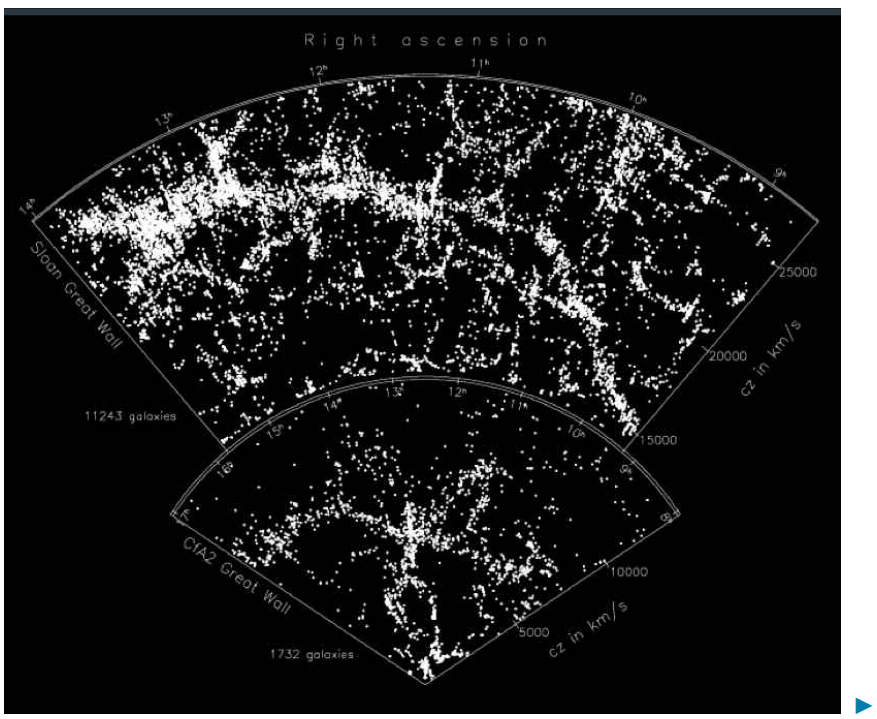




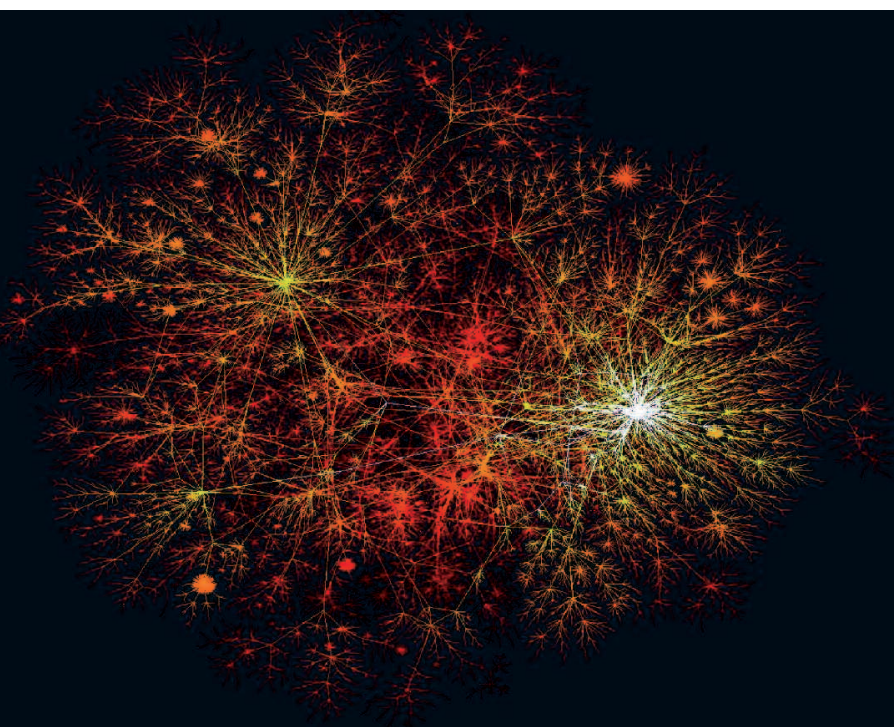

$\Delta$ FIG. 4: Complex vs. complicated: Internet network structure. A complicated object is a computer for which there is always a detailed top down project. For Internet the situation is different, the links are added bottom up and there is no global project. This we call complex.

\section{The science of complexity today}

The study of complex systems refers to the emergence of collective properties in systems with a large number of parts in interaction among them. These elements can be atoms or macromolecules in a physical or biological context, but also people, machines or companies in a socio-economic context. The science of complexity tries to discover the nature of the emerging behaviour of complex systems, often invisible to the traditional approach, by focusing on the structure of the interconnections and the general architecture of systems, rather than on the individual components.

It is a change of perspective in the forma mentis of scientists rather than a new scientific discipline. Traditional science is based on a reductionistic reasoning for which, if one knows the basic elements of a system, it is possible to predict its behaviour and properties. It is easy to realize, however, that for a cell or for the socio-economic dynamics one faces a new situation in which the knowledge of the individual parts is not sufficient to describe the global behaviour of the structure. Starting from the simplest physical systems, like critical phenomena in which order and disorder compete, these emergent behaviour $s$ can be identified in many other systems, from ecology to the immunitary system, to the social behaviour and economics. The science of complexity has the objective of understanding the properties of these systems. Which rules govern their behaviour? How do they adapt to changing conditions? How do they learn efficiently and how do they optimize their behaviour?

The development of the science of complexity cannot be reduced to a single theoretical or technological innovation: It implies a novel scientific approach with enormous potentialities to deeply influence the scientific activities, social, economic and technological.

\section{Statistical Mechanics}

The science of complexity arises naturally from statistical mechanics which, in the seventies, introduced a fundamental change of paradigm with respect to the reductionistic scientific vision. At the equilibrium point between order and disorder one can observe fluctuations at all scales; the system cannot be described any more with the usual formalism in which one tries to write simple equations for average quantities. From this conceptual grain many new concepts have developed which produced a revolution in our way of looking at nature: scaling laws, renormalization group, fractal geometry, glassy and granular systems, complex liquids, colloids and many others. More recently it begins to be clear that these concepts can have much broader applications with respect to the physical systems from which they originated. This led to a large number of interdisciplinary applications which are sometimes surprising and which probably represent just the beginning of the many possible applications.

\section{Complexity in the science of new materials}

Superconductivity, which is a sort of historical paradigm of complex collective behaviour, developed in the last years into the new field of high temperature superconductivity. It represents one of the most challenging open problems in science. In addition, the development of new materials, like for example the new carbon compounds, the electronic bio-molecular materials, the nanostructured meta-materials and photonic crystals require a radically new approach for their preparation and understanding. Their formation corresponds in fact to a dissipative dynamics far from equilibrium with possible elements of self-organization. This perspective has been described in the paper "Basic Research in the Information Technology Industry" in Physics Today (July 2003, p.44-49) from T.N. Theis and P.M. Horn (IBM labs). In this paper it is argued that dissipative systems, hierarchy of energy scales, critical self-organization and inspiration from evolutive biological systems will be fundamental for the development of the new nanomaterials.

\section{FIG. 5: Wrapped piece of paper.}

A very simple process, wrapping a piece of paper, leads to a complex fractal structure with dimension $D=2.5$. This conceptually powerful example shows that highly complex structures may be generated by very simple dynamical processes.

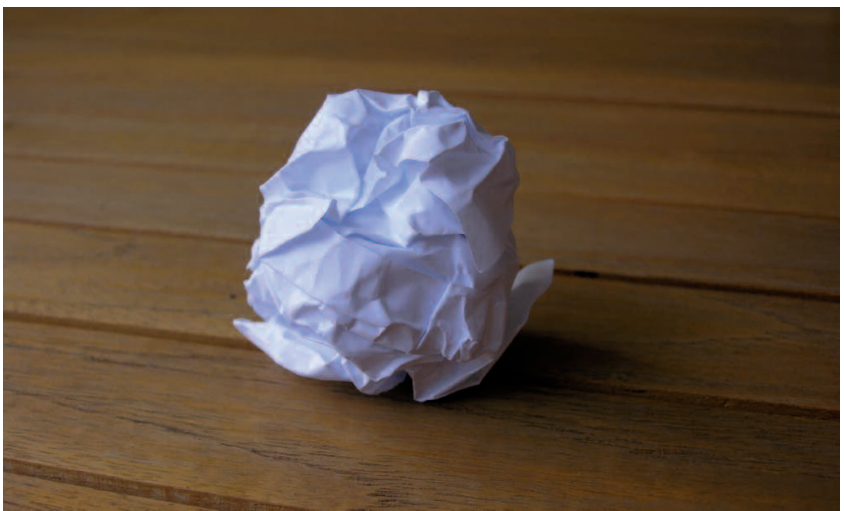




\section{Self-organization at work}

An important example of the above scenario is the development of complex networks like Internet and the World-Wide-Web, which represent today the basic substrate for all sort of communications, personal, business and military at the planetary level. These networks have the remarkable property that they have not been designed by anybody! The usual "top down" approach to the engineering of a telephone or a satellite communication system has been replaced by a "bottom up" dynamics in which new servers are added to the networks or are eliminated. This type of dynamics presents properties of self-organization and biological evolution which require a completely new and interdisciplinary vision.

\section{The search for relevant information}

A characteristic feature of our society, for both the scientific and the socio-economic activities, is the necessity of finding one's way in the enormous amount of information available, in order to extract the relevant information. This is an epochal problem which requires the development of an information theory with innovative characteristics in which the theory of complexity and critical phenomena can play a crucial role. An example of this situation is given by the Google search engine which uses concepts from statistical physics to explore the WEB and for the hierarchical categorization of the information. At the moment Google is the best search engine for the quality of the ordering of information, and there is great activity to improve it and to define the new generation. Most likely this will require a new interdisciplinary effort in which linguistic and semantic ideas are also included in the optimization process.

The examples mentioned describe some typical situations in which an interdisciplinary approach based on the concepts and methods of the Science of Complexity appear particularly relevant. One could easily add many more and the situation is highly dynamical. In fact, it would be easy to speculate about the potential of these methods in scientific areas which have not yet been explored from this perspective. 